apresentaçāo de caso

\author{
Antônio Carlos Bonaccorsi
}

Instituto Estadual de Diabetes e Endocrinologia (IEDE), Rio de Janeiro, $R J$.

Recebido em 05/11/99

Revisado em 26/04/00 e 17/07/00

Aceito em 25/07/00

\section{Insuficiência Androgênica de Origem Hipotálamo-Hipofisária em Dois Homens com Disfunção Sexual}

\section{RESUMO}

Existem homens não idosos com disfunção sexual que apresentam niveis subnormais de testosterona sérica e niveis normais ou baixos de prolactina e gonadotropinas, sem anormalidade anatômica demonstrável em hipófise ou hipotálamo. Esta situação é considerada uma forma de hipogonadismo hipogonadotrópico idiopático, possivelmente de origem hipotalâmica. Estamos apresentando e discutindo o quadro clínico de dois pacientes com uma disfunção similar, nos quais a disfunção sexual precedeu o aparecimento do hipoandrogenismo. Nossa conclusão é que o hipoandrogenismo foi conseqüência de um distúrbio psiconeuroendócrino devido à disfunção sexual. (Arq Bras Endocrinol Metab 2000;44/5: 440-444)

Unitermos: Hipogonadismo hipogonadotrópico; Disfunção sexual; Hipoandrogenismo; Stress; Clomifeno

\begin{abstract}
There is a group of middle-aged men with sexual dysfunction that presents reduced serum testosterone levels and normal to low levels of prolactin and gonadotropins, but without any demonstrable hypothalamicpituitary anatomic abnormality. This condition is considered a form of idiopathic hypogonadotropic hypogonadism, probably of hypothalamic origin. We present and discuss two patients with similar features in whom sexual dysfunction preceded actual hypoandrogenism. We conclude that in these two patients hypoandrogenism was the consequence of a psychoneuroendocrine disturbance due to the sexual dysfunction. (Arq Bras Endocrinol Metab 2000;44/5: 440-444)
\end{abstract}

Keywords: Hypogonadotropic hypogonadism; Sexual dysfunction; Hypoandrogenism; Stress; Clomiphene

A ausas endócrinas são bem comprovadas em cerca de $10 \%$ dos casos de disfunção sexual (DS) $(1,2)$. No entanto, têm sido descritos casos de pacientes não idosos com DS e hipoandrogenismo secundário na ausência de lesão orgânica hipotálamo-hipofisária, nos quais a origem destes achados permanece obscura. Estamos apresentando os casos de dois destes pacientes, e discutindo as possíveis causas do hipoandrogenismo e sua relação com a DS.

\section{Caso 1}

Paciente de 46 anos, casado e com um filho de 5 anos, visto em abril de 1997 com libido normal e queixas de disfunção erétil e ejaculação precoce. Sendo professor universitário em fase de doutoramento, encontrava-se sob stress intenso (sic), que era agravado pela própria DS. Não fumava, mas referia ingestão diária de bebidas alcoólicas. Seu IMC era $21 \mathrm{~kg} / \mathrm{m}^{2}$. Os exames clínico e da genitália resultaram normais. A capacidade erétil era normal, comprovada por um teste de injeção intracavernosa de prostaglandina. 
$\mathrm{Na}$ época tinha testosterona total (TT) de $316 \mathrm{ng} / \mathrm{dl}$, testosterona livre (TL) de 14,2pg/ml, LH de $1,6 \mathrm{UI} / \mathrm{L}$ e FSH de $0,8 \mathrm{UI} / \mathrm{L}$.

Por apresentar certo grau de depressão foi medicado com um antidepressivo fitoterápico (hypericum, $600 \mathrm{mg} / \mathrm{d}$ ) o qual, com a afirmação de ausência de disfunção endócrina, produziu nítida melhora clínica. $O$ paciente voltou à consulta em junho de 98 com as mesmas queixas, tendo sido prescrito Sildenafil $50 \mathrm{mg}$, com o qual seu temor de desempenho se reduziu muito, passando a ter relações sexuais normais.

Face a resposta subnormal durante um teste do LHRH (com TT, TL e prolactina - tabela 1), foi solicitada tomografia computadorizada (TC) de crânio e sela turca, que foi considerada normal, ainda que um discutivel nódulo hipodenso à esquerda determinasse discreto abaulamento do contorno superior da hipófise.

Iniciado, então, citrato de Clomifeno $(25 \mathrm{mg} / \mathrm{d})$, sendo nova avaliação hormonal realizada após 60 dias, notando-se normalização dos níveis androgênicos. Após este período e ainda em uso de Sildenafil, o paciente referia melhora da libido. Com a normalização dos níveis androgênicos, foi orientado a permanecer só com Sildenafil e hypericum.

Voltou em julho de 1999 para nova avaliação hormonal (usando apenas Sildenafil), que revelou TT de 239ng/dl, TL de 4,9pg/ml, Prl de 4,2ng/ml, FSH de $0,7 \mathrm{UI} / \mathrm{L}$ e $\mathrm{LH}$ de $1,9 \mathrm{UI} / \mathrm{L}$. Como o paciente estava de partida para o exterior, foi solicitada uma ressonância magnética (RM) que mostrou sela turca normal. Foi então prescrito Clomifeno $(50 \mathrm{mg}$ em dias alternados) por 60 dias, aos quais se seguiriam períodos de descanso de 60 dias, e assim por diante, até seu retorno dentro de 12 meses.

\section{Caso 2}

Paciente de 37 anos, divorciado e sem filhos, visto em 1990 com queixas de desinteresse sexual e temor de desempenho. Notava-se um componente obsessivo de perfeição em seu trabalho e nas relações interpessoais e sexuais, o que o mantinha sob stress. Não fumava, nem usava bebidas alcóolicas, drogas ou qualquer medicação. Os exames físico e da genitália eram normais. Seu IMC era $22,8 \mathrm{~kg} / \mathrm{m}^{2}$.

$\mathrm{Na}$ época tinha TT de $700 \mathrm{ng} / \mathrm{dl}$ e $\mathrm{Prl}$ de $6,2 \mathrm{ng} / \mathrm{ml}$. Após ser submetido a um teste de ereção com prostaglandina intracavernosa, que resultou normal, foi-lhe aconselhado psicoterapia, que foi recusada.

Reavaliado em 1993, apresentava TT de $280 \mathrm{ng} / \mathrm{dl}$, TL de $10,5 \mathrm{pg} / \mathrm{ml}$ e $P r l$ de $12,5 \mathrm{ng} / \mathrm{ml}$. Foi-lhe prescrito tamoxifeno $(20 \mathrm{mg} / \mathrm{d})$ por 30 dias, ao fim dos quais tinha TT de 420ng/dl, TL de $20,7 \mathrm{pg} / \mathrm{ml}$, LH de $3,5 \mathrm{UI} / \mathrm{L}$ e Prl de $3,6 \mathrm{ng} / \mathrm{ml}$. Nova avaliação, após 30 dias do término do tamoxifeno, mostrava TT de 195ng/dl, TL de 11,8pg/ml, LH de $3,6 \mathrm{UI} / \mathrm{L}$ e FSH de 1,9UI/L. Visando observar o efeito de um androgênio sobre a DS do paciente, foithe prescrito undecanoato de testosterona (40mg VO a cada $8 \mathrm{hs}$ ) por 3 meses, ao fim dos quais o quadro clínico manteve-se inalterado.

Voltou a ser visto 4 anos mais tarde, em novo casamento e com 2 filhas de 1 e 3 anos, referindo desinteresse sexual e perdas ocasionais de ereção durante o ato sexual. Fez nova avaliação androgênica com teste de LHRH (100mcg IV) e teste do Clomifeno (100mg/d por 10 dias).

Não tendo ocorrido resposta normal ao Clomifeno, foi realizado um teste de reserva androgênica testicular com hormônio gonadotrópico coriônico (HCG, em dose única de 5.000UI) com o qual, 72hs após sua aplicação, a TT subiu para $416 \mathrm{ng} / \mathrm{dl}$ e a TL para $20,5 \mathrm{pg} / \mathrm{ml}$, demonstrando função normal das células de Leydig. Uma TC de crânio e sela turca também resultou normal. Face à ausência de doença orgânica detectável, foi novamente aconselhada terapia sexual, a qual foi recusada. Por este motivo, foi prescrito ao casal o uso de injeções

Tabela 1. Niveis plasmáticos de testosterona total (Ti), livre (TL), prolactina (Prl) e respostas do FSH e $\mathrm{LH}$ aos testes de LHRH e Clomifeno realizados nos pacientes 1 e 2 .

\begin{tabular}{|c|c|c|c|c|c|c|c|c|c|}
\hline \multirow[t]{3}{*}{ Pac. } & \multirow{3}{*}{$\begin{array}{l}\text { T Total } \\
\text { (ng/dl) }\end{array}$} & \multirow{3}{*}{$\begin{array}{c}\text { T livre } \\
(\mathrm{pg} / \mathrm{ml})\end{array}$} & \multirow{3}{*}{$\begin{array}{l}\mathrm{Prl} \\
(\mathrm{ng} / \mathrm{ml})\end{array}$} & \multirow{2}{*}{\multicolumn{4}{|c|}{ Teste LHRH }} & \multirow{2}{*}{\multicolumn{2}{|c|}{$14(11<1)$}} \\
\hline & & & & & & & & & \\
\hline & & & & Basal & $30^{\prime}$ & $60^{\prime}$ & Basal & $30^{\prime}$ & $60^{\prime}$ \\
\hline $\begin{array}{l}1 \\
2\end{array}$ & $\begin{array}{l}272 \\
225\end{array}$ & $\begin{array}{l}8,5 \\
8.8\end{array}$ & $\begin{array}{r}7.7 \\
10,8\end{array}$ & $\begin{array}{l}0,6 \\
1,7\end{array}$ & $\begin{array}{l}0,7 \\
3,0\end{array}$ & $\begin{array}{l}0,8 \\
3,4\end{array}$ & $\begin{array}{l}0,8 \\
3,0\end{array}$ & $\begin{array}{l}8,0 \\
15,1\end{array}$ & $\begin{array}{l}8.2 \\
14.4\end{array}$ \\
\hline $\begin{array}{l}1\left(^{(*)}\right) \\
2\left(^{* *}\right) \\
2\left(^{* * *}\right)\end{array}$ & $\begin{array}{l}750 \\
288 \\
605\end{array}$ & $\begin{array}{l}22,7 \\
10,5 \\
10,7\end{array}$ & - & $\begin{array}{l}1,1 \\
2,0 \\
3,5\end{array}$ & & & & $\begin{array}{l}3.8 \\
4.7 \\
4,5\end{array}$ & \\
\hline V.N. & $247-827$ & $8,7-54,2$ & $2-11,5$ & $1-10,5$ & & & & $1-8,4$ & \\
\hline
\end{tabular}

(*) Clomifeno, $25 \mathrm{mg} / \mathrm{d}$ por 60 dias

$\left(^{\star *}\right)$ Clomifeno, $100 \mathrm{mg} / \mathrm{d}$ por 10 dias

$\left(^{* * *}\right.$ Clomifeno, $50 \mathrm{mg}$ em dias alternados por 60 dias 
intracavernosas de prostaglandina, que vem sendo utilizado ocasionalmente, até hoje, com pleno sucesso e nítido alívio da DS.

A avaliação periódica dos níveis androgênicos mostrou: TT de $270 \mathrm{ng} / \mathrm{dl}$ e TL de $11,0 \mathrm{pg} / \mathrm{ml}$, no início de 1998 e, TT de $278 \mathrm{ng} / \mathrm{dl}$, TL de $5,4 \mathrm{pg} / \mathrm{ml}$, LH de $3,7 \mathrm{UI} / \mathrm{L}$ e $\operatorname{Prl}$ de $1,6 \mathrm{ng} / \mathrm{ml}$, em 1999, quando uma RM de crânio e sela turca resultou normal. Um estímulo com Clomifeno (50mg em dias alternados) por 60 dias, não resultou em alterações favoráveis sobre a função sexual.

Apesar do escasso resultado terapêutico da normalização androgênica, manteve-se o mesmo esquema de Clomifeno (50mg em dias alternados) por 2 meses, seguindo-se idênticos periodos de descanso, e assim por diante, devendo ser revisto dentro de 1 ano.

\section{DISCUSSĀO}

Têm sido descritos casos de homens com DS, nos quais coexistem niveis subnormais de testosterona e normais de gonadotropinas e $\mathrm{Prl}$, não sendo encontradas anomalias anatômicas hipotálamo-hipofisárias que justifiquem tal fato (3). O quadro laboratorial destes pacientes configura um hipogonadismo secundário e, mais precisamente, um hipogonadismo hipogonadotrópico idiopático pós-puberal $(3,4)$. A resposta ao teste de $\mathrm{LHRH}$ é normal mas, inesperadamente, mais lenta do que seria de se esperar em presença de níveis androgênicos subnormais. Já a resposta ao Clomifeno é normal e o seu uso em baixas doses por longo prazo leva à normalização androgênica (5). O conjunto destes achados laboratoriais indica uma possível deficiência na liberação de LHRH (3-5), cuja causa poderia ser qualquer uma das que levam a uma redução dos níveis de testosterona, tais como stress $(6,7)$, abuso de álcool (8), doenças sistêmicas crônicas ou agudas (9), idade avançada e outras $(8,10,11)$.

Em nossos dois pacientes, o stress seria a única presente, dentre as causas citadas. Em ambos, plenamente saudáveis do ponto de vista clínico, coexistiam níveis androgênicos permanentemente reduzidos $\mathrm{e}$ gonadotropinas normais baixas ou subnormais, não havendo lesão orgânica hipotálamo-hipofisária ou hiperprolactinemia. $O$ caso 1 mostrou resposta deficiente e o caso 2 resposta normal ao estímulo pelo LHRH. No que se refere ao teste do Clomifeno, o paciente 1 , submetido a um longo período de estímulo, respondeu normalmente. Já no paciente 2 não houve resposta ao estímulo clássico de $100 \mathrm{mg}$ diários por 10 dias. No entanto, respondeu normalmente a um estímulo mais prolongado, o que parece indicar uma possível disfunção do centro hipotalâmico gerador de LHRH como a causa do problema.

Esta disfunção seria semelhante à que ocorre no homem idoso, e dever-se-ia à liberação de uma massa menor de LHRH a cada pulso, o que levaria à redução do número de pulsos espontâneos de LH de maior amplitude. Isto resultaria em possível menor liberação de LH biológico, como comprovado em homens idosos impotentes (12) e saudáveis (13), ainda que, posteriormente, contestada por Kaufman e col. (14).

Não sendo idosos, esta insuficiência hipotálamo-hipofisária em presença de stress psicossocial em nossos pacientes dever-se-ia, provavelmente, a uma disfunção psiconeuroendócrina e poderia ser explicada pela endocrinologia comportamental a qual, ao estudar a interação entre hormônios e comportamento, conclui que esta interação é bidirecional: os hormônios afetando o comportamento e vice-versa (15). Estudo de Nilsson e col. (16) parece comprovar esta suposição: analisando os efeitos adversos do stress psicossocial na função gonadal $\mathrm{e}$ nos níveis de insulina de 39 homens com mais de 51 anos, concluíram que o stress pode estar associado a um processo de envelhecimento prematuro em homens não idosos, que se traduziria por uma insuficiência testicular e sinais de resistência insulínica, resultado de regulação neuroendócrina negativa do stress sobre a função gonadal. Estes efeitos danosos do stress seriam indiretos (via hipotálamo-hipofisária) ou diretos sobre os testículos.

Neurotransmissores (NT) como a serotonina, as catecolaminas, a dopamina, o acido gamaaminobutírico e a histamina e neuropeptídeos (NP) como a galanina, neuropeptídeo Y (NPY) e opióides endógenos, entre outros, agem conjuntamente sobre o eixo hipotálamo-hipófise-testicular a nível central (6,7,17-19) e, muitos deles, também a nível testicular (20). Redução dos níveis hipotalâmicos de NPY pode estar implicada na disfunção do centro regulador de LHRH e no conseqüente hipoandrogenismo do homem idoso (17).

Dentre os NP, os opióides endógenos têm sido dos mais estudados na sua relação com stress e disfunção reprodutiva e sexual $(6,18)$. Considerando que o stress psicológico crônico leva a uma maior liberação de catecolaminas e cortisol a nível periférico e central de opióides endógenos com conseqüente inibição da liberação de LHRH, alem de indução de hiperprolactinemia, é muito provável que estas alterações levem a uma insuficiência androgênica em determinados pacientes com DS. 
Em ratos submetidos a stress, foi comprovada uma diminuição da síntese de $\mathrm{T}$ nos testículos devida, provavelmente, a uma hipossensibilidade das células de Leydig às gonadotropinas e ao bloqueio pós-AMPc da síntese (6). Assim, é possível que uma disfunção destes NP e NT relacionada ao stress, à idade e a outros fatores desconhecidos possa levar, tanto a nível central como periférico, à ocorrência de uma insuficiência androgênica. Nos dois pacientes apresentados, é notável a ausência de hipoandrogenismo laboratorial a época das primeiras queixas de DS, vindo a mesma a se manifestar posteriormente. Isto indicaria que o hipoandrogenismo seria conseqüência e não causa da DS, o que é explicado pelos atuais conhecimentos da endocrinologia comportamental (15) e caracterizado como um distúrbio psiconeuroendócrino.

A normalização dos níveis androgênicos não produziu efeitos positivos nítidos na vida sexual de nossos pacientes, parecendo indicar que a DS não estava relacionada ao hipoandrogenismo ou, então, que além dos androgênios, outros fatores funcionais estariam implicados. Um destes fatores, as endorfinas, estando elevadas durante o stress crônico e naquele decorrente de temor do desempenho coital, teriam ação inibitória sobre a avaliação dos estímulos sexuais pelos centros límbico-mesencefálicos, o que manteria a DS mesmo após reposição hormonal (18). Deve-se notar que quando a função erétil destes pacientes foi restabelecida pelo uso do Sildenafil e das injeções intracavernosas de prostaglandina, o temor de desempenho se reduziu a níveis mínimos com nítida melhora da satisfação sexual.

Sempre que indicada, a reposição hormonal deve ser realizada visando confirmar a sempre possível correlação entre o hipoandrogenismo e a DS destes pacientes. Como terapia inicial, recomenda-se o Clomifeno em baixas doses e por períodos mais prolongados, já que os pacientes são responsivos ao mesmo (5). Mais estudos são necessários e desejáveis sobre hipoandrogenismo e sexualidade. No momento atual, ainda não sabemos se nestes pacientes a insuficiência androgênica é causa ou conseqüência da DS, ou mesmo se há alguma relação com ela e de que maneira $(5,10)$. No entanto, há indícios que a DS antecedeu o aparecimento do hipoandrogenismo no quadro clínico dos dois pacientes aqui apresentados.

\section{REFERÊNCIAS}

1. Nichel JC, Morales A, Candra M, Fenemore J, Sunridge $\mathrm{DH}$. Endocrine dysfunction in impotence: incidence. significance and cost-effective screening. J Urol 1984: 132:40-3.

2. Bonaccorsi AC, Faria Ji R. Niveis de testosterona total e livre e aspectos clínicos em 154 homens com disfunção sexual. Arq Bras Endocrinol Metab 1997:41:173-6.

3. Guay AT, Bansal S, Hodge MB. Possible hypothalamic impotence. Male counterpart to hypothalamic amenorrea? Urology 1991:38:317-22.

4. Spark RF. Neuroendocrinology and impotence. Ann Intern Med 1983;98:103-5.

5. Guay AT, Bansal S, Heatley GJ, Effect of raising endogenous testosterone levels in impotent men with secondary hypogonadism: Double blind placebo-controlled trial with Clomiphene citrate J Clin Endocrinol Metab 1995;80:3546-53.

6. Collu R, Gibb W, Ducharne JR. Effects of stress on the gonadal function. J Endocrinol Invest 1984;7:529-37.

7. Nilsson PM, Moller L, Solstad K. Adverse effects of psychosocial stress on gonadal function and insulin levels in middle-aged males, J Intern Med 1995;237:479-86.

8. Kaufman JM, Vermeulen A. Androgens in male senescence. In: Nieschlag E, Behre HM, eds. Testosterone. Berlin: Springer: 1998:437-71.

9. Handelsman DJ. Testicular dysfunction in systemic disease. Endocrinol Metab Clin North Am 1994:23:839-56.

10. Koreman SG, Morley JE, Moorandian AD, Davis SS, Kaiser $\mathrm{FE}$, et al. Secondary hypogonadism in older men: its relation to impotence. J Clin Endocrinol Metab 1990;71:963-9.

11. Vermeulen A. Clinical review 24. Androgens in the aging male. J Clin Endocrinol Metab 1991;73:221-4.

12. Nankin HR, Calkins JH. Decreased bioavailable testosterone in aging normal and impotent men. J Clin Endocrinol Metab 1986;63:1418-22.

13. Urban RJ, Veldhuis JD, Blizzard RM, Dufau ML. Attenuated release of biologically active luteinizing hormone in healthy aging men. J Clin Invest 1988:81:1020-9.

14. Kaufman JM, Giri M, Deslypere JM, Thomas $G$, Vermeulen A. Influence of age on the responsiveness of the gonadotrophs to luteinizing hormone-releasing hormone in males. J Clin Endocrinol Metab 1991;72:1255-60.

15. Christiansen K. Behavioural correlates of testosterone. In: Nieschlag E, Behre HM, eds. Testosterone. Berlin: Springer; 1998:107-42.

16. Nilsson PM, Moller L, Solstad K. Adverse effects of psychosocial stress on gonadal function and insulin levels in middle-aged males. J Intern Med 1995;237:479-86.

17. Kalra SP, Sahu A, Crowley WR, Kalra PS, Neuropeptidergic control of gonadotropin secretion: facilitation by androgens. In: Serio M, ed. Perspectives in Andrology. New York: Raven Press: 1989: 139-48

18. Fabbri A, Jannini EA, Gnessi L, Moretti C, Ulisse S, et al Endorphins in male impotence: Evidence for naltrexone stimulation of erectile activity in patient therapy. Psychoneuroendocrinology 1989:14:103-11.

19. Negro-Villar A, Lopez F, Merchenthaler I, Chin M, Valenca M, et al. Pituitary-testicular interactions: role of $\mathrm{LH}-\mathrm{RH}$, 
inhibin, steroids and intra-cellular messengers. In Isidori A, Fabbri A, Dufau ML, eds. Hormonal Communicating

Events in The Testis. New York: Raven Press; 1990:87-94.

20. Fabbri A, Ulisse S, Bolotti M, Ridolfi M, Spera $G$, et al. Opioid regulation of testicular function. In: Serio $M$, ed. Perspectives in Andrology. New York: Raven Press; 1989:203-14.

\section{Endereço para correspondência:}

Antônio Carlos Bonaccorsi

Av. Nossa Senhora de Copacabana 647, sala 1115 22.050-000 Rio de Janeiro, RJ 